\title{
Accidents de travail impliquant des jeunes Canadiens : analyse de 22 années de surveillance des données recueillies à partir du Système canadien hospitalier d'information et de recherche en prévention des traumatismes
}

\author{
B. Pratt, M.H.P. (1, 2); J. Cheesman (1); C. Breslin, Ph. D. (3); M. T. Do, Ph. D. (1, 2)
}

Cet article a fait l'objet d'une évaluation par les pairs.

Diffuser cet article sur Twitter

Résumé

Introduction : L'inexpérience, une formation inadéquate et une exposition accrue au danger sont susceptibles de contribuer à un risque plus élevé d'accidents chez les jeunes travailleurs. Cette étude décrit les caractéristiques des accidents de travail impliquant de jeunes Canadiens afin d'identifier les secteurs pour lesquels élaborer des stratégies de prévention en matière d'accidents de travail.

Méthodologie : Nous avons analysé le dossier des jeunes de 10 à 17 ans qui se sont présentés à un service des urgences (SU) faisant partie du Système canadien hospitalier d'information et de recherche en prévention des traumatismes (SCHIRPT) entre 1991 et 2012. Nous avons classé les accidents de travail en fonction de groupes d'emploi correspondant à la Classification nationale des professions (codes statistiques 2006) et nous avons effectué des analyses descriptives afin d'établir le profil des accidents en fonction de ces groupes d'emploi. Nous avons calculé des rapports proportionnels de blessures (RPB) en fonction de l'âge et du sexe ainsi que des intervalles de confiance à $95 \%$ pour comparer la nature des blessures survenues en contexte professionnel et en contexte non professionnel, à la fois dans l'ensemble et par groupe d'emploi.

Résultats : Parmi les 6046 blessures ayant eu lieu au travail $(0,72 \%$ des cas dans ce groupe d’âge), 63,9 \% touchaient des garçons. Les jeunes (54,6 \% de garçons) œuvrant dans l'industrie des aliments et des boissons ont formé $35,4 \%$ des consultations aux SU en lien avec le travail et 10,2 \% des admissions liées au travail, alors que les travailleurs du secteur primaire $(76,4 \%$ de garçons) représentaient 4,8 \% des consultations aux SU liées au travail et 24,6\% des admissions liées au travail. Les RPB ont été significativement élevés pour les brûlures $(9,77$, IC à $95 \%: 8,94$ à 10,67), les blessures par écrasement et les amputations $(6,72$, IC à $95 \%: 5,79$ à 7,80), les blessures causées par l'électricité (6,04, IC à $95 \%: 3,64$ à 10,00), les morsures (5,09, IC à $95 \%: 4,47$ à 5,79), les plaies ouvertes $(2,68$, IC à $95 \%: 2,59$ à 2,78) et les lésions oculaires (2,50, IC à $95 \%: 2,20$ à 2,83) dans un contexte professionnel par rapport aux cas en contexte non professionnel. Ceci s'explique en bonne partie par l'incidence proportionnelle élevée de certains types de blessures spécifiques à des groupes d'emploi.

Conclusion : Nos conclusions fournissent des renseignements relatifs à certains groupes professionnels sur des types de blessures courantes susceptibles de favoriser l'adoption d'approches ciblées à l'égard de la réduction de l'incidence des blessures chez les jeunes en milieu de travail.

Mots-clés : adolescent, surveillance des blessures, accidents de travail, jeunes travailleurs, blessures chez les jeunes

\section{Points saillants}

- Les données sur les blessures de jeunes qui se présentent aux services des urgences d'onze hôpitaux pédiatriques et de six hôpitaux généraux au Canada sont enregistrés dans le Système canadien hospitalier d'information et de recherche en prévention des traumatismes (SCHIRPT).

- Nous avons utilisé les données du SCHIRPT de 1991 à 2012 pour découvrir comment les jeunes travailleurs de 10 à 17 ans ont été blessés au travail.

- Les jeunes qui travaillaient dans l'industrie des aliments et des boissons ont constitué la majorité des victimes d'accident de travail s'étant présentées aux services des urgences d'un hôpital.

- Les admissions à un hôpital attribuables à des accidents de travail ont concerné principalement des jeunes travaillant dans certaines professions (construction/réparation, machinerie/travail des métaux/travail électrique et travail non qualifié), dans le secteur manufacturier et dans le secteur primaire.

- Les jeunes travailleurs de l'industrie des aliments et des boissons et de la construction ont connu une incidence proportionnelle beaucoup plus élevée de brûlures, alors que les livreurs et les travailleurs dans les services à l'enfance ont présenté plus souvent des morsures et que les lésions oculaires et les blessures par écrasement ou amputations ont été davantage courantes chez les travailleurs qualifiés, les manutentionnaires et les conducteurs de véhicules et les travailleurs du secteur primaire.

- Par rapport aux accidents non liés au travail, nous avons constaté des variations de l'incidence de plusieurs types de blessures, notamment de morsures et de brûlures, en fonction de l'âge parmi les jeunes travailleurs.

Rattachement des auteurs :

1. Division de la surveillance et de l'épidémiologie, Agence de la santé publique du Canada, Ottawa (Ontario), Canada

2. École de santé publique Dalla Lana, Université de Toronto, Toronto (Ontario), Canada

3. Institut de recherche sur le travail et la santé, Toronto (Ontario), Canada

Correspondance : Minh T. Do, Division de la surveillance et de l'épidémiologie, Agence de la santé publique du Canada, 785, avenue Carling, Ottawa (Ontario), K1A 0K9; courriel : Minh.T.Do@phac-aspc.gc.ca 


\section{Introduction}

De nombreux jeunes obtiennent un emploi pour les bénéfices intrinsèques et matériels que celui-ci leur procure : acquérir des compétences et gagner de l'argent ${ }^{1}$. En 2014, près de la moitié des jeunes de 15 à 19 ans, soit environ 840000 Canadiens, étaient présents sur le marché du travail ${ }^{2}$. Cependant, le risque de blessures au sein de ce groupe d'âge annule souvent les avantages du travail. Entre 2011 et 2013, les Commissions des accidents du travail (CAT) du Canada ont enregistré 20 décès parmi les travailleurs de 15 à 19 ans $^{3}$ et 23996 réclamations concernant des accidents avec perte de temps ont été acceptées dans ce seul groupe d'âge ${ }^{4}$. Même si les données sur les blessures professionnelles pour les travailleurs de moins de 15 ans sont rares, les sondages menés auprès d'élèves d'école secondaire américains et canadiens ont révélé que $18 \%$ des travailleurs de 10 à 14 ans ont signalé des accidents de travail survenus dans le cadre d'un emploi d'été ${ }^{5}$ et 49,7\% des salariés de 12 à 14 ans ont signalé des accidents de travail survenus au cours de l'année précédente ${ }^{6}$.

Les invalidités attribuables aux blessures qui se produisent tôt dans la vie professionnelle peuvent avoir des effets sur les capacités professionnelles futures et des effets à long terme sur la santé7. Les jeunes ayant subi des accidents de travail font plus de visites annuelles chez le médecin généraliste que ceux qui n’en ont pas subi ${ }^{8}$. Parmi les diverses catégories professionnelles, ce sont les jeunes œuvrant comme travailleurs manuels et dans le secteur de la production de biens qui courent davantage de risques de subir des blessures entraînant une absence pour invalidité professionnelle ou une augmentation du nombre de réclamations auprès de la CAT pour accidents avec perte de travail, par rapport aux jeunes qui travaillent dans le secteur des services $^{7,9}$.

Même si les données sur les réclamations sont instructives, elles ne portent pas sur les blessures de jeunes travailleurs qui ne sont pas couverts par la CAT ou ne sont pas admissibles par elle. Puisque les jeunes sont plus susceptibles d'occuper un emploi temporaire, occasionnel, contractuel ou saisonnier que les adultes ${ }^{10}$ et puisque les jeunes travailleurs occupent souvent des emplois informels ou autonomes qui ne sont pas obligatoirement ou efficacement couverts dans toutes les provinces ${ }^{11}$, comme c'est le cas dans le secteur agricole, le gardiennage ou certaines entreprises familiales ${ }^{5}$, les données sur les réclamations sont susceptibles de sousestimer les blessures chez les jeunes. Ce phénomène est accentué par la sous-déclaration générale d'accidents de travail par les jeunes employés, qui craignent des représailles, se sentent impuissants ou croient que leur employeur ne se souciera pas de leurs préoccupations, ou encore parce qu'ils ne sont pas au courant de la manière d'effecteur une déclaration $^{12-14}$.

La fréquence et la distribution des accidents de travail chez les jeunes ont été décrites à l'aide de données recueillies à partir du Système canadien hospitalier d'information et de recherche sur la prévention des traumatismes (SCHIRPT) dans un article paru en $2005^{15}$. Les établissements de surveillance des services des urgences (SU) participant à ce système ont saisi une partie élargie de la « base » de la pyramide des blessures, en intégrant des événements qui n'étaient pas assez graves pour entraîner une hospitalisation, et indépendamment de la couverture des CAT ou de la réception des réclamations. De plus, les données du SCHIRPT constituent une source abondante de renseignements sur les caractéristiques cliniques et fournissent une description détaillée des circonstances entourant l'accident.

Depuis la publication originale d'il y a dix ans, notre analyse mise à jour tire profit d'une augmentation de l'efficacité statistique, en raison du plus grand nombre de cas saisis au fil du temps, et comprend des analyses comparatives des caractéristiques des blessures liées au travail et des blessures non liées au travail.

Étant donné le poids important des accidents de travail chez les jeunes, la détermination des caractéristiques des blessures et des facteurs de risque spécifiques à cette population a des répercussions sur l'amélioration de la santé de la main-d'œuvre actuelle et future ainsi que sur la prévention d'une utilisation prolongée des soins de santé liés à des accidents de travail. À l'aide de données du
SCHIRPT sur 22 ans, nous avons décrit les caractéristiques individuelles et professionnelles liées aux accidents de travail impliquant des jeunes, en établissant des comparaisons avec les blessures non liées au travail et entre les groupes d'emploi.

\section{Méthodologie}

\section{Source des données}

Le SCHIRPT, système de surveillance des blessures fondé sur les SU sous la responsabilité de l'Agence de la santé publique du Canada, a été lancé en 1990. Les établissements participants recueillent des informations détaillées sur les blessures et les empoisonnements auprès des SU de 11 hôpitaux pédiatriques et de 6 hôpitaux généraux au Canada. Ces renseignements sont consignés dans un formulaire de rapport du SCHIRPT constitué d'une page remplie par le patient (ou en son nom) qui décrit les circonstances entourant la blessure et d'une page remplie par le médecin qui fournit les caractéristiques cliniques de la blessure. Le codage des formulaires, centralisé, est réalisé par des employés qualifiés pour cette tâche à l'Agence de la santé publique du Canada. Depuis 2010, la saisie des entrées dans le formulaire de rapport est électronique (e-SCHIRPT) : les formulaires sont remplis directement par les coordonnateurs des établissements par le biais d'une application Web centrale.

Les données recueillies par le SCHIRPT ont déjà été décrites et évaluées comme étant de bonne qualité lorsqu'elles sont prudemment utilisées à des fins de recherche en santé publique ${ }^{16}$.

\section{Population à l'étude}

Nous avons inclus dans notre analyse les jeunes de 10 à 17 ans ayant rempli un formulaire du SCHIRPT entre le $1^{\text {er }}$ janvier 1991 et le 31 décembre 2012. Nous avons choisi comme âge limite supérieur 18 ans car c'est l'âge en deçà duquel des restrictions législatives relatives à l'emploi au Canada s'appliquent ${ }^{6}$. La limite inférieure, 10 ans, a été sélectionnée afin de consigner des données sur les jeunes qui déclarent des conditions de travail informelles ${ }^{5}$. Les 14 dossiers avec une date de naissance 
manquante ont été éliminés lors de l'extraction de données initiale.

\section{Classification des accidents de travail}

Les accidents de travail ont été recensés, et ce, aussi bien dans la version imprimée que sur le formulaire de l'e-SCHIRPT, à partir d'une autodéclaration, au moyen d'une question à choix de réponses oui/non selon laquelle le participant indiquait s'il « travaillait contre rémunération » au moment de la blessure. Comme les formulaires imprimés du SCHIRPT remplis avant 2011 contenaient des questions à propos du contexte dans lequel la blessure s'était produite, les dossiers où le contexte a été codé " en service » ont aussi été sélectionnés comme accidents de travail. Les descriptions d'accidents faites par les participants ont été revues, et l'emploi occupé au moment de la blessure a été codé à partir des 11 catégories de la Classification nationale des professions (codes des statistiques 2006), en fonction du niveau de compétence et des tâches exécutées (http:// www.statcan.gc.ca/fra/sujets/norme/cnp/ 2006/cnp2006-menu) ${ }^{17}$. Si la profession n'a pas pu être établie à partir de la description seule (p. ex. coupure accidentelle au travail), le lieu où la blessure s'est produite a été utilisé pour la déterminer (p. ex. cuisine de restaurant).

Même si les professions dans l'armée sont très variées et peuvent être classées d'une façon similaire aux professions civiles ${ }^{17}$, en raison de l'environnement organisationnel uniforme de ces blessures, elles ont été constituées en groupe distinct d'emploi, celui de l’armée en général.

\section{Caractéristiques des blessures}

Les caractéristiques des blessures ont été déterminées à partir de la nature de la blessure (NB) « la plus grave » inscrite par le médecin traitant. Étant donné les fréquences faibles associées à certains types de blessures, celles-ci ont été regroupées en fonction d'une matrice tenant compte de la similitude entre les types de blessures ou les parties du corps touchées, en intégrant la possibilité d'une classification erronée. Les blessures musculo-squelettiques regroupaient les entorses ou foulures, les blessures à des muscles ou à des tendons, les blessures des tissus mous non spécifiées ailleurs et les subluxations du coude ${ }^{19}$.

\section{Analyse statistique}

Nous avons calculé les distributions descriptives des fréquences pour les données catégorielles et les écarts moyens ou écartstypes (ET) pour les caractéristiques continues (individuelles et des blessures), selon s'il s'agissait d'un accident de travail ou d'une blessure non liée au travail, et en fonction du groupe d'emploi et de l'admission à l'hôpital. Nous avons calculé les rapports proportionnels des blessures (RPB) pour comparer l'occurrence relative des NB entre les jeunes blessés dans un contexte de travail et ceux blessés dans un autre contexte, pour l'ensemble, par groupe d'emploi et par catégorie d'âge ${ }^{20}$. Le RPB est le rapport des cas observés en fonction de l'âge et du sexe (groupes d'âge de 1 an pour les calculs globaux et deux catégories, moins de 15 ans et 15 ans et plus, pour les calculs en fonction du groupe d'âge) dans le sous-groupe des blessés en contexte professionnel par rapport aux cas prévus selon l'incidence dans le groupe des blessés en contexte non professionnel. Le RPB a été calculé pour toutes les catégories de $\mathrm{NB}$, regroupées selon des intervalles de confiance (IC) à $95 \%$ pour en déterminer la signification statistique ${ }^{21}$. Un RPB supérieur à 1 indique que la NB observée dans le sous-groupe des blessés en contexte professionnel est plus courante que prévu, en fonction de la NB dans le groupe de référence des blessés hors contexte professionnel. Le RPB a été utilisé dans des études antérieures ${ }^{22-24}$ et constitue une mesure utile pour évaluer la fréquence des accidents lorsque les renseignements sur les dénominateurs de la population ne sont pas accessibles $^{25}$.

Toutes les analyses statistiques ont été effectuées à l'aide de la version $9.4 \mathrm{du}$ logiciel SAS pour Windows ${ }^{26}$ et Microsoft Excel 2010.

\section{Résultats}

Nous avons récupéré au départ 835888 dossiers de blessures chez les 10 à 17 ans entre 1991 et 2012 à partir des formulaires imprimés et des formulaires de l'e-SCHIRPT. Nous avons exclu 167 dossiers qui ne signalaient pas le sexe du jeune blessé. Parmi les 5983 dossiers codés comme étant des accidents « de travail " et les 102 dossiers indiquant que la blessure s'était produite « en service » (à partir de la variable « contexte ", " travail " codé comme 0 avant 2011), 7 dossiers ont été retirés parce qu'ils étaient probablement des doublons de dossiers d'un seul accident et 32 autres dossiers ont été recodés comme n'étant pas liés au travail car, selon la description, la blessure ne s'était pas produite au travail ni dans un environnement y ressemblant (p. ex. « blessé tandis qu'il suivait des cours de théâtre dans une école d'art dramatique »). La réponse « oui » à la question dichotomique relative au travail figurant sur le formulaire du SCHIRPT a été sélectionnée dans 14 dossiers pour lesquels, selon les descriptions, les jeunes faisaient du bénévolat. Cependant, nous les avons conservés comme accidents de travail dans les analyses en raison de la diversité des emplois informels occupés par les jeunes, qui ne leur donnent peut-être pas droit à une paye mais qui sont similaires à des emplois rémunérés en ce qui concerne les tâches, l'environnement et l'exposition au risque ${ }^{5,6}$.

La population finale à l'étude était de 6046 accidents de travail et 829668 accidents non liés au travail. Puisque très peu de blessures entraînant le décès à la suite d'une consultation à des SU ont été saisies dans nos données (143, soit moins de 0,02\%) et qu'aucune d'entre elles n'était liée à un accident de travail, nous les avons conservées dans notre analyse avec les accidents non liés au travail utilisés pour générer les proportions de référence.

Le tableau 1 présente les caractéristiques des blessures stratifiées en fonction du sexe par situation professionnelle. Les garçons étaient concernés dans la majorité des accidents de travail comme des accidents non liés au travail $(63,9 \%$ et $61,7 \%)$, et les jeunes blessés au travail avaient, en moyenne, environ 2,5 ans de plus que les jeunes blessés ailleurs qu'au travail. Les jeunes hommes de 17 ans représentaient le quart des jeunes ayant subi des blessures au travail, et les jeunes de moins de 12 ans composaient moins de $3 \%(n=173) d u$ total. Les cinq NB les plus courantes (plaies ouvertes, blessures musculo-squelettiques, plaies superficielles, fractures et brûlures) 
TABLEAU 1

Caractéristiques des blessures de jeunes recensées dans le SCHIRPT, au travail et hors travail, 1991 à 2012

\begin{tabular}{|c|c|c|c|c|c|c|}
\hline \multirow[t]{2}{*}{ Caractéristiques } & \multicolumn{3}{|c|}{ Accident de travail } & \multicolumn{3}{|c|}{ Blessure non liée au travail } \\
\hline & Tous & Garçons & Filles & Tous & Garçons & Filles \\
\hline Nombre total (et \%) & $6046(0,72)$ & $3861(63,9)$ & $2185(36,1)$ & $829668(99,28)$ & $511662(61,7)$ & $318006(38,3)$ \\
\hline \multicolumn{7}{|l|}{ Nature de la blessure (et \%) } \\
\hline Plaies ouvertes & $1951(32,3)$ & $1408(36,5)$ & $543(24,9)$ & $90629(10,9)$ & $64861(12,7)$ & $25768(8,1)$ \\
\hline Fractures/dislocations & $538(8,9)$ & $350(9,1)$ & $188(8,6)$ & $225707(27,2)$ & $153128(29,9)$ & $72579(22,8)$ \\
\hline Brûlures/corrosion & $448(7,4)$ & $223(5,8)$ & $225(10,3)$ & $5469(0,7)$ & $3064(0,6)$ & $2405(0,8)$ \\
\hline Lésions oculaires & $225(3,7)$ & $169(4,4)$ & $56(2,6)$ & $12504(1,5)$ & $8537(1,7)$ & $3967(1,2)$ \\
\hline Blessures à la tête & $201(3,3)$ & $112(2,9)$ & $89(4,1)$ & $58856(7,1)$ & $39577(7,7)$ & $19279(6,1)$ \\
\hline Empoisonnement & $34(0,6)$ & $26(0,7)$ & $8(0,4)$ & $19301(2,3)$ & $6177(1,2)$ & $13124(4,1)$ \\
\hline Multiples/dentaires/autres & $24(0,4)$ & $17(0,4)$ & $7(0,3)$ & $9063(1,1)$ & $6472(1,3)$ & $2591(0,8)$ \\
\hline Blessure causée par l'électricité & $15(0,2)$ & $9(0,2)$ & $6(0,3)$ & $327(0)$ & $183(0,0)$ & $144(0,0)$ \\
\hline Engelure/stress dû à la chaleur ou au froid/surmenage systémique & $11(0,2)$ & a & a & $755(0,1)$ & $494(0,1)$ & $261(0,1)$ \\
\hline Noyade/immersion ou asphyxie & $9(0,1)$ & a & a & $904(0,1)$ & $512(0,1)$ & $392(0,1)$ \\
\hline Manquant, non spécifié ou non décelé & $111(1,8)$ & $64(1,7)$ & $47(2,2)$ & $16560(2)$ & $9803(1,9)$ & $6757(2,1)$ \\
\hline Admission à l'hôpital (et \%) & $170(2,8)$ & $138(3,6)$ & $32(1,5)$ & $44902(5,4)$ & $29382(5,7)$ & $15520(4,9)$ \\
\hline
\end{tabular}

Abréviations : SCHIRPT, Système canadien hospitalier d'information et de recherche en prévention des traumatismes.

${ }^{a}$ Valeur supprimée, 5 observations ou moins chez un sexe ou les deux.

formaient $83 \%$ de l'ensemble des accidents de travail. Les jeunes avec des blessures non liées au travail étaient plus fréquemment admis à l'hôpital que ceux ayant déclaré des accidents de travail $(5,4 \%$ contre $2,8 \%$ ), et les garçons étaient plus fréquemment admis à l'hôpital dans les deux groupes. Les garçons et les filles ont subi des types de blessures différents : $36,5 \%$ des garçons blessés au travail ont eu des plaies ouvertes contre $24,9 \%$ des filles, et $28,1 \%$ des filles blessées au travail ont présenté des blessures musculosquelettiques contre $19 \%$ des garçons. Nous avons observé des motifs similaires de blessures en fonction du sexe pour les blessures liées au travail et non liées au travail, même si leur ampleur était différente.

Le tableau 2 montre les distributions par sexe et par groupe d'âge des accidents de travail et des admissions à l'hôpital par groupe d'emploi. Parmi les visites aux SU attribuables à des accidents de travail,
$35,4 \%(n=2141)$ concernaient des jeunes occupant un emploi dans l'industrie des aliments et des boissons (54,6 \% de garçons, $\mathrm{n}=1168$ ). Les garçons avaient le pourcentage le plus élevé de blessures parmi les métiers (construction/réparation, machinerie/travail des métaux/travail en électricité et travail non qualifié), comme manutentionnaires ou conducteurs de véhicules et comme travailleurs du secteur primaire (exploitation forestière/foresterie, exploitation minière, pêche et agriculture), alors que les accidents de travail chez les filles étaient surtout en lien avec des emplois de soutien dans le domaine des services et des loisirs. La contribution relative de chaque groupe d'emploi aux consultations aux SU et à l'admission subséquente à l'hôpital a varié de façon importante : les jeunes blessés dans le groupe d'emploi ayant la plus grande proportion de consultations aux SU liées au travail (travailleurs de l'industrie des aliments et des boissons) ne représentaient que 10,2 \% des admissions liées au travail, alors que les travailleurs du secteur primaire $(76,4 \%$ de garçons) représentaient seulement 4,8 \% des consultations aux SU liées au travail mais 24,6 \% des admissions liées au travail.

Nous avons aussi observé des différences en fonction des groupes d'âge (moins de 15 ans et 15 ans et plus) dans les groupes d'emploi (tableau 2). Les jeunes plus âgés ont formé la majorité des consultations aux SU pour des accidents de travail dans la plupart des groupes. Cependant, les travailleurs de soutien dans les services à l'enfance et les préposés aux soins personnels ainsi que les travailleurs du secteur primaire de 10 à 14 ans représentaient une proportion relativement élevée des consultations aux liés au SCHIRPT pour ces groupes d'emploi (respectivement $40,4 \%$ et $30,2 \%)$. Le groupe d'emploi des livreurs était la seule catégorie où la majorité était composée des travailleurs les plus jeunes $(75,5 \%$ étaient âgés de moins de 15 ans). 


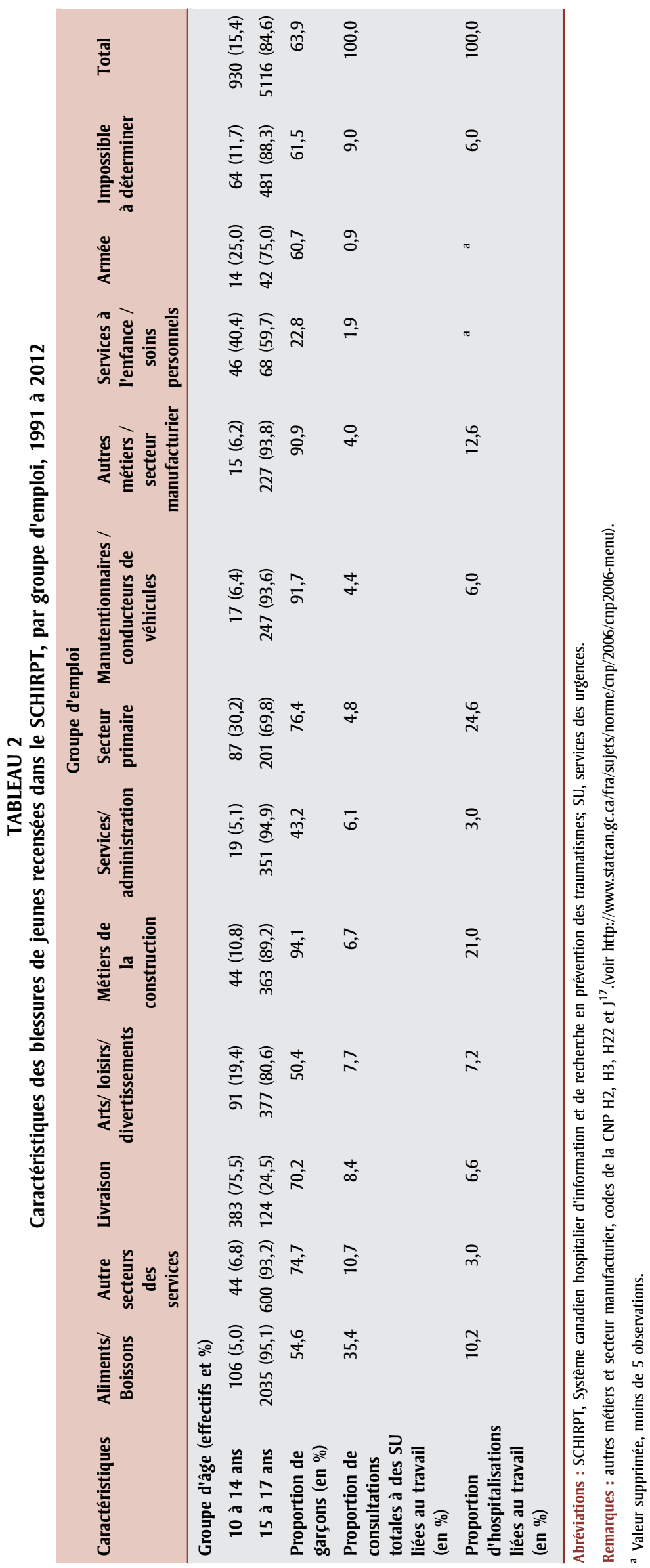

Par rapport aux blessures non liées au travail, nous avons observé des RPB significativement élevés pour ce qui est des blessures liées au travail concernant des plaies ouvertes $(\mathrm{RPB}=2,68$, IC à $95 \%$ : $2,59$ à 2,78$)$, des brûlures $(9,77$, IC à $95 \%$ : 8,94 à 10,67), des lésions oculaires (2,50, IC à $95 \%: 2,20$ à 2,83), des morsures $(5,09$, IC à $95 \%: 4,47$ à 5,79), des blessures par écrasement et des amputations (6,72, IC à $95 \%: 5,79$ à 7,80$)$ et des blessures causées par l'électricité $(6,04$, IC à $95 \%$ : 3,64 à 10,00) (tableau 3). Même si la plupart des RPB stratifiés en fonction de l'âge n'ont pas semblé différer de façon significative (c.-à-d. qu'il n'y a pas eu chevauchement des IC), les adolescents plus âgés ont affiché un RPB supérieur pour les brûlures par rapport aux jeunes de 10 à 14 ans $(\mathrm{RPB}=10,53$, IC à $95 \%: 9,62$ à 11,53 et RPB = 4,38, IC à $95 \%$ : 2,98 à 6,43 respectivement), et les jeunes âgés de 10 à 14 ans avaient un RPB supérieur pour les morsures par rapport à celui de jeunes plus âgés (RPB $=18,48$, IC à $95 \%: 15,75$ à 21,69 et RPB $=2,20$, IC à $95 \%: 1,74$ à 2,78 respectivement) (tableau 3).

Par rapport au groupe d'emploi, les travailleurs de l'industrie des aliments et des boissons (RPB $=24,36$, IC à $95 \%: 22,28$ à 26,62) et les travailleurs de métier ou les travailleurs du secteur manufacturier ont subi davantage de blessures que les jeunes blessés en dehors du travail. De même, les livreurs $(\mathrm{RPB}=36,97$, IC à $95 \%$ : 32,23 à 42,41) et les travailleurs dans les services à l'enfance et préposés aux soins personnels $(\mathrm{RPB}=29,27$, IC à $95 \%: 21,08$ à 40,63) avaient une incidence de morsures plus élevée que prévu (tableau 4). Les blessures par écrasement ou amputation avaient des RPB constamment élevés dans l'ensemble des groupes d'emploi des travailleurs manuels et de l'industrie produisant des biens, particulièrement chez les travailleurs qualifiés et du secteur manufacturier $(\mathrm{RPB}=22,68$, IC à $95 \%: 15,34$ à 33,54$)$. Ce groupe, qui comprend des travailleurs des métaux, comme des soudeurs, avait aussi l'incidence proportionnelle la plus élevée de lésions oculaires $(\mathrm{RPB}=13,01, \mathrm{IC}$ à $95 \%: 10,11$ à 16,74). Même si les blessures à la tête ne se sont pas produites plus souvent que prévu dans l'ensemble des accidents de travail, les jeunes œuvrant dans le domaine des arts, des loisirs et du divertissement ont connu 
TABLEAU 3

Rapports proportionnels des blessures (RPB) ajustés par âge et par sexe : nature des blessures liées au travail chez les jeunes par rapport aux blessures non liées au travail, par groupe d'âge, 1991 à 2012

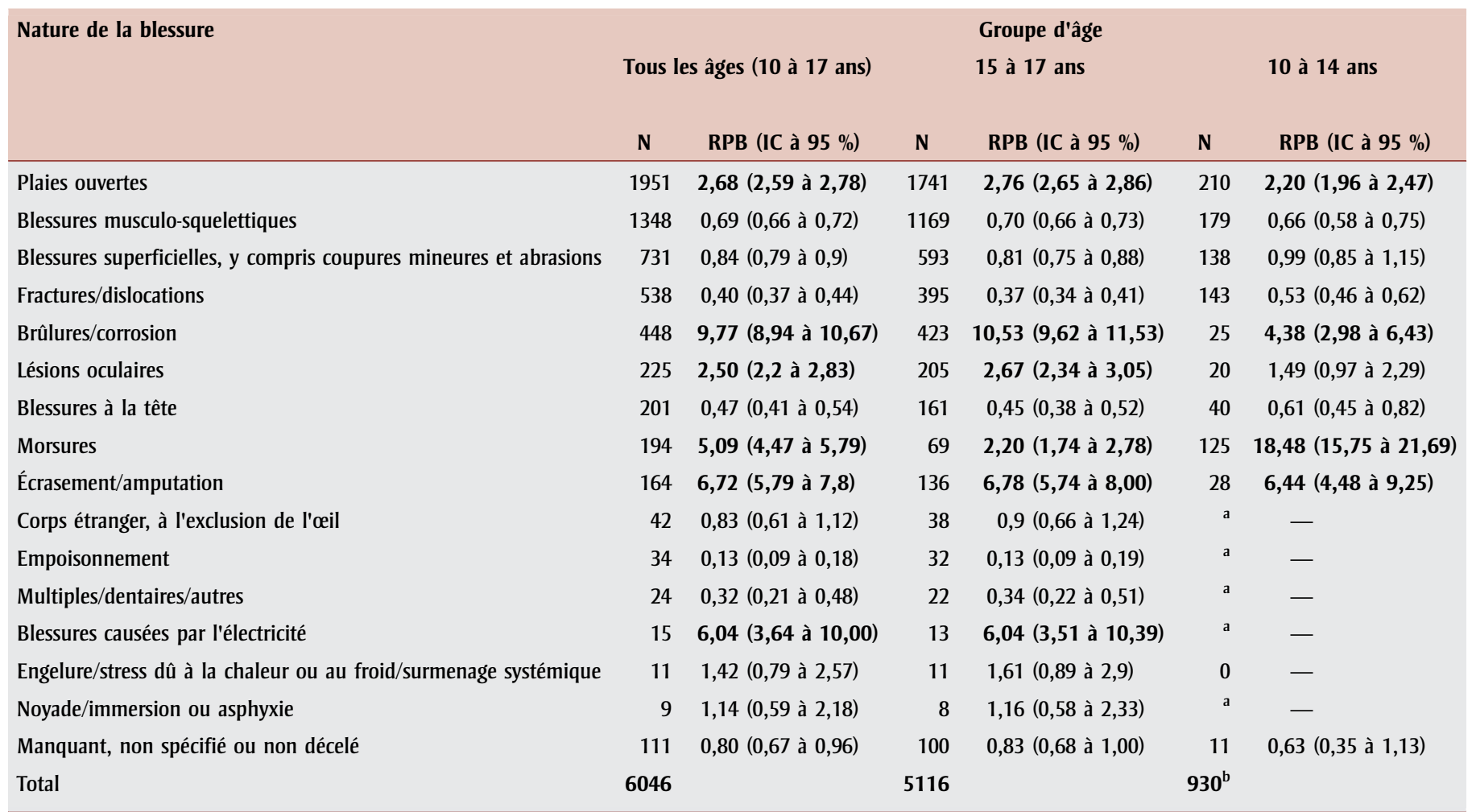

Abréviations : IC, intervalle de confiance; NB, nature de la blessure; RPB, rapports proportionnels des blessures.

Remarque : les valeurs en gras indiquent des RPB significativement élevés, limite inférieure de I'IC à 95 \% supérieure à 1.

${ }^{a}$ Le RPB n'est pas présenté pour la NB lorsque $\mathrm{N}<5$.

b Le total comprend les catégories de NB lorsque $\mathrm{N}<5$.

une incidence significativement supérieure de blessures à la tête par rapport aux jeunes ayant subi des blessures non liées au travail $(\mathrm{RPB}=1,34$, IC à $95 \%: 1,01$ à 1,77$)$ (tableau 4).

Le tableau 5 montre les blessures les plus courantes au sein de chaque groupe d'emploi. Les blessures par écrasement ou amputation n'ont touché que moins de $3 \%$ des jeunes ayant consulté des SU liés au SCHIRPT en lien avec le travail, mais elles étaient plus courantes dans le secteur primaire et chez les manutentionnaires/conducteurs de véhicules et représentaient près du cinquième des admissions liées au travail ( $n=29,17,1 \%$; données non présentées). Les blessures musculo-squelettiques étaient courantes chez les jeunes travailleurs du secteur des services et les manutentionnaires, même si elles ne se produisaient pas plus fréquemment dans le cadre d'accidents de travail que d'accidents non liés au travail, comme le reflètent les RPB. D'autres blessures, comme les plaies ouvertes et les brûlures chez les travailleurs de l'industrie des aliments et des boissons et les morsures chez les livreurs, étaient courantes et significativement élevées parmi les jeunes ayant subi des accidents de travail par rapport aux autres jeunes.

\section{Analyse}

Notre étude permet de conclure que les jeunes ayant déclaré des accidents de travail à des SU étaient principalement des garçons, ce que reflètent les distributions caractéristiques des types d'emploi en fonction du sexe et ce qui est conforme à la littérature existante. La plupart des blessures se sont produites dans des emplois du secteur des services, particulièrement dans celui des aliments et des boissons, même si les blessures chez les travailleurs qualifiés, les travailleurs du secteur manufacturier et les travailleurs du secteur primaire ont plus souvent entraîné une admission à l'hôpital. Nous avons aussi montré que, mis à part le fait qu'ils ont connu dans l'ensemble une incidence proportionnelle élevée de brûlures, de blessures par écrasement ou amputation, de morsures, de blessures causées par l'électricité, de lésions oculaires et de plaies ouvertes, les jeunes avaient des incidences proportionnelles significativement plus élevées de types de blessures particuliers, spécifiques à chaque groupe d'emploi et à chaque catégorie d'âge.

Dans une étude sur les accidents de travail en fonction des groupes d'âge, les adolescents avaient un taux d'invalidité permanent découlant d'amputation, de brûlures et de coupures supérieur à celui des adultes? . Cette occurrence s'est reflétée dans nos données, où les plaies ouvertes (dont les coupures les plus graves), les brûlures et les blessures par écrasement ou amputation représentaient environ la moitié des accidents de travail. De même, d'après les conclusions d'une étude américaine récente qui a analysé les dossiers des SU, les travailleurs âgés de 15 à 19 ans ont subi deux fois plus de brûlures que les travailleurs 


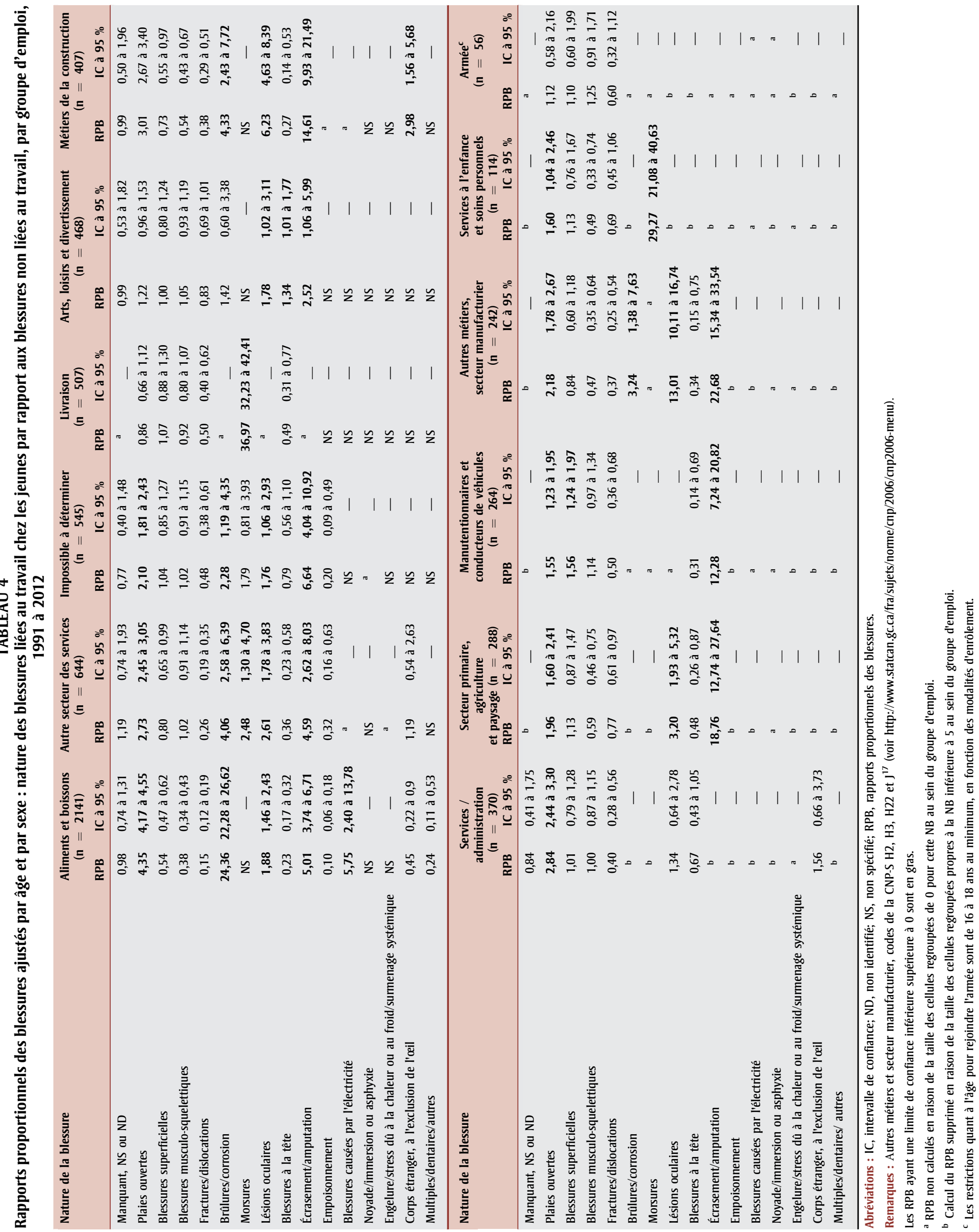




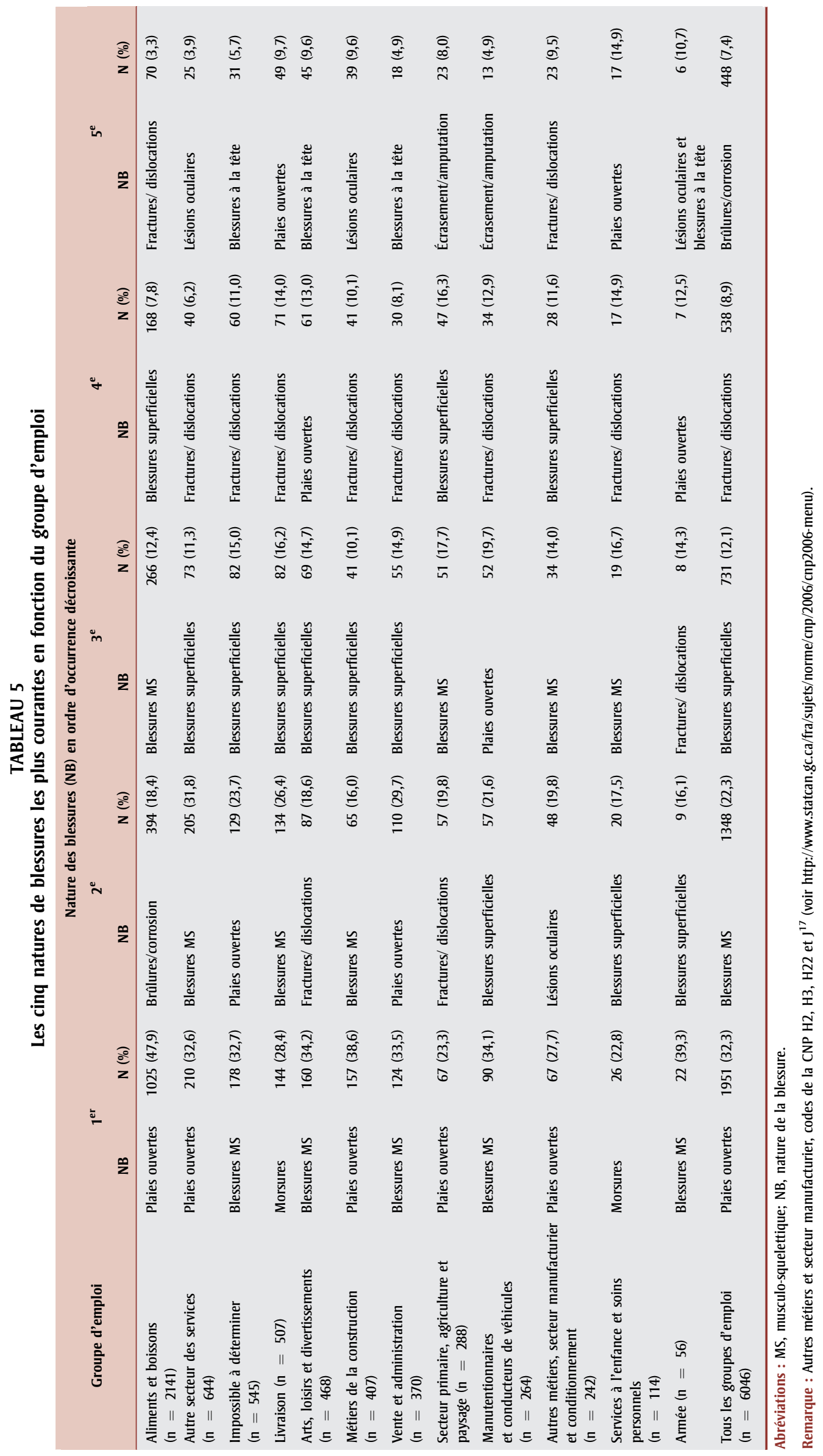


plus âgés ${ }^{27}$ et, comme dans notre étude, les brûlures étaient plus courantes parmi les travailleurs dans les services d'hébergement et de restauration, du secteur agricole, du secteur manufacturier et de la construction. Nous avons observé un RPB particulièrement élevé pour les brûlures chez les travailleurs adolescents plus âgés de l'industrie des aliments et des boissons et, puisque seulement 26,1 \% des travailleurs adolescents exposés à des risques de blessures déclarent utiliser une protection contre les brûlures (p. ex. des gants de cuisine ${ }^{28}$, certaines stratégies visant à réduire les obstacles à l'utilisation d'un équipement de protection seraient utiles pour réduire les causes primaires de blessures chez les jeunes dans ces emplois courants.

Les lésions oculaires et les blessures par écrasement ou amputation étaient presque systématiquement supérieures dans tous les groupes d'âge par rapport aux accidents non liés au travail, particulièrement dans les emplois spécialisés, du secteur manufacturier et du secteur primaire. Les études précédentes sur les lésions oculaires chez les travailleurs de la construction adultes ont attribué cela en partie à une protection oculaire inadéquate $e^{29}$. Même si nous n'avons pas enquêté sur l'utilisation d'équipement de protection individuelle dans notre étude, on peut penser que les jeunes travailleurs perçoivent moins les risques de lésions oculaires et sont moins susceptibles d'utiliser une protection oculaire $^{30}$. Il convient de noter que, étant donné l'augmentation de la possibilité d'invalidité permanente ${ }^{7}$, les RPB élevés et la fréquence élevée d'hospitalisation pour des blessures par écrasement ou amputation sont la preuve qu'il est important de trouver des mesures de protection efficaces pour les jeunes exposés à des dangers au travail. Notre observation selon laquelle le RPB pour ce type de blessure était égal chez les jeunes de 10 à 14 ans et chez les adolescents plus âgés indique que les jeunes travailleurs dans certains environnements de travail sont susceptibles d'être exposés à des conditions, à de l'équipement ou à des tâches qui ne correspondent pas à leur niveau de compétence.

Les RPB faibles pour les blessures musculosquelettiques sont le reflet des taux inférieurs de blessures liées à des foulures répétitives chez les jeunes travailleurs par rapport aux travailleurs plus âgés ${ }^{31}$.
Cependant, malgré leur incidence relativement faible, il s'agissait d'accidents de travail tout de même courants chez les jeunes dans notre étude, particulièrement chez les filles. Étant donné la probabilité d'une utilisation persistante ou prolongée de soins de santé ${ }^{8}$, ainsi que d'une augmentation du nombre de blessures musculosquelettiques liées au travail qui se produisent avec l'âge, nos conclusions donnent du poids aux recommandations selon lesquelles la priorité doit être accordée aux efforts de prévention des blessures tôt dans la vie professionnelle ${ }^{7}$.

Soulignons aussi que nous avons observé un RPB supérieur pour les blessures à la tête chez les jeunes travailleurs du domaine des arts, des loisirs et du divertissement qui n'est pas ressorti dans les RPB regroupés. Ces travailleurs étaient principalement des conseillers de camp, des sauveteurs et d'autres animateurs ou moniteurs en loisirs. Cette spécificité des blessures reflète sans doute leur similitude contextuelle par rapport aux blessures liées aux sports, qui comptent habituellement une grande proportion de blessures à la tête chez les jeunes ${ }^{32}$. Les traumatismes cérébraux légers subis à un jeune âge ayant été associés à un niveau d'études et à une situation d'emploi inférieurs $^{33}$, les conséquences de ces blessures sont susceptibles d'être de longue durée.

L'hétérogénéité des blessures en fonction des emplois soulève la question de la détermination des meilleures façons de protéger les jeunes travailleurs. Un sondage mené auprès de travailleurs ontariens de 14 à 18 ans a révélé que, même si plus de $90 \%$ d'entre eux avaient reçu une formation en matière de sécurité, $38 \%$ étaient sans supervision pour au moins une partie de la journée de travail ${ }^{11}$. Bien que nous n'ayons pas été en mesure d'évaluer l'état de la supervision dans notre étude, les jeunes travaillant à titre de moniteurs en loisirs ou d'éducateurs de la petite enfance (p. ex. gardiens d'enfants) ou dans la livraison sont souvent eux-mêmes superviseurs ou travaillent seuls. Notre observation de RPB significativement élevés pour les blessures à la tête (travailleurs du domaine des arts et loisirs) et les morsures (techniciens de services à l'enfance et livreurs) dans ces groupes peut être une indication de l'absence inhérente de supervision. C'est particulièrement évident à la lumière du jeune âge de nombre de ces travailleurs. Dans de tels cas, il est sans doute plus réaliste d'insister sur l'utilisation d'équipement de sécurité et de renforcer la formation avant le début de l'emploi.

\section{Points forts et limites}

Nous avons mené une étude approfondie en élargissant l'analyse descriptive de base grâce à l'application de RPB en fonction de l'âge et du sexe, avec lesquels nous avons été en mesure d'évaluer statistiquement les différences dans l'incidence de NB entre les sousgroupes liés aux accidents de travail et une population de référence avec blessures non liées au travail. Les RPB ont été comparés avec les blessures non liées au travail et ils dépendent donc de la distribution des blessures dans cette population de référence, ce qui fait qu'ils ne peuvent pas être interprétés comme des taux. Ils sont néanmoins instructifs en ce qu'ils sont susceptibles de fournir des indices par rapport à des mécanismes sous-jacents dans les tendances touchant les blessures observées. Si l'on utilise les blessures à la tête comme exemple, les RPB plutôt faibles donnent à penser que, en plus du fait que les blessures à la tête sont plus courantes dans le cadre de blessures non liées au travail, il est possible que certaines mesures visant à prévenir les blessures à la tête chez les jeunes travailleurs dans certains emplois, par exemple grâce à un casque de protection sur les chantiers, puissent conférer un effet protecteur. La comparaison de l'incidence des accidents de travail par rapport aux accidents non liés au travail à l'aide du RPB nous permet de tenir compte de facteurs contextuels spécifiques aux environnements professionnels entraînant des tendances ou des types de blessures différents.

Nous avons appliqué le système de classification national des professions normalisé (CNP-S) à nos données afin de présenter un portrait nuancé des emplois que les jeunes occupaient lorsqu'ils ont été blessés. L'utilisation de ce mécanisme permet la comparaison avec des statistiques nationales recueillies à partir d'autres sources de données, comme l’Enquête sur la population active $^{34}$. L'uniformité marquée entre nos résultats et ceux publiés dans la littérature prouve que les données sur l'emploi des 
jeunes tirées des dossiers du SCHIRPT codés à l'aide des lignes directrices de la CNP-S se comparent à celles d'autres études enquêtant sur les accidents de travail de jeunes utilisant d'autres critères. Même si ce mécanisme de classification empêche l'attribution d'une incidence de blessure à des emplois particuliers, les groupes de la CNP permettent de classer ensemble des emplois qui supposent des tâches des fonctions, des exigences en matière d'éducation et des environnements comparables. Les emplois faisant partie d'un même groupe sont susceptibles d'exposer les travailleurs à des types de dangers similaires. Cette méthode a cependant une limite : il n'y a pas d'algorithme normalisé pour ce qui est de l'attribution d'emplois ou de tâches.

Notre étude comporte aussi plusieurs limites, lesquelles peuvent influer sur les résultats. La plupart des SU liés au SCHIRPT ne sont pas représentatifs de la population, car ce sont des postes sentinelles situés stratégiquement pour exercer une surveillance active. Certains groupes sont sousreprésentés, en particulier les adolescents âgés et les résidents de régions rurales ou éloignées, ce qui fait que les types de blessures et les groupes d'emploi propres à ces régions et à ces populations sont susceptibles d'être également sous-estimés dans notre analyse. Les blessures nécessitant une admission sont également davantage susceptibles d'être moins importantes dans les établissements visés par le SCHIRPT, ce qui fait que nos estimations sous-évaluent probablement la fréquence de blessures plus graves chez les jeunes ${ }^{16}$. Il reste que les données caractéristiques des blessures recueillies à partir de plusieurs établissements faisant partie du SCHIRPT et présumés comme étant pleinement représentatifs de la population ont révélé une corrélation élevée avec les données recueillies à partir d'une enquête nationale axée sur la population. Les données des établissements faisant partie du SCHIRPT représentatifs de la population étaient aussi conformes à celles d'autres établissements faisant partie du SCHIRPT ${ }^{35}$. Cela donne à penser que la validité externe des données sur les blessures du SCHIRPT peut être déduite en restant prudent, afin qu'il soit possible d'évaluer les tendances nationales agrégées des blessures chez les jeunes ${ }^{35}$. De plus, une étude portant sur les blessures attribuables aux sports chez les jeunes a révélé une validité élevée des caractéristiques des blessures saisies par un hôpital pour enfants du SCHIRPT par rapport à celles saisies par une source de données administrative locale ${ }^{36}$. Le pourcentage de garçons de 10 à 17 ans se présentant à un établissement visé par le SCHIRPT était similaire à celui des jeunes hommes ayant subi un traumatisme saisi par le Système national d'information sur les soins ambulatoires (SNISA) $(61,7 \% \text { et } 59,2 \%)^{37}$, et le fait que le groupe signalé par le SNISA soit légèrement plus âgé (de 10 à 19 ans) peut sans doute expliquer cette petite différence.

Au moment de l'extraction des données, la transition entre les dossiers imprimés du SCHIRPT et l'e-SCHIRPT était en cours, ce qui fait que les données de 2011 et de 2012 n'étaient pas complètes pour tous les établissements. Pour évaluer la sensibilité des caractéristiques du groupe d'emploi lors de l'inclusion aux dossiers de l'e-SCHIRPT à partir d'années incomplètes, la proportion d'accidents de travail pour chaque groupe d'emploi a été calculée à l'aide des dossiers imprimés complets seulement pour les blessures s'étant produites entre 1991 et 2010, puis de nouveau pour l'ensemble complet de données. Les proportions d'accidents de travail par groupe d'emploi n'ont pas semblé avoir été touchées de façon importante par l'exhaustivité de l'entrée des dossiers annuels, car le changement de pourcentage entre les deux calculs était inférieur à $10 \%(-5,10 \%$ à 5,66 \%) pour chaque groupe (résultats non présentés). De même, les analyses n'utilisant que les dossiers d'années ayant une entrée de données complète (1991-2010) ont produit des RPB différents négligeables, sans qu'il y ait de changement dans les niveaux de signification (chevauchement des IC) par rapport aux analyses utilisant tous les dossiers jusqu'à 2012. Par exemple, les RPB et les IC à $95 \%$ pour les plaies ouvertes de 1991 à 2010 et de 1991 à 2012 étaient de 2,64 $(2,52$ à 2,76$)$ et de $2,68 \quad(2,57$ à 2,80$)$ respectivement (résultats non présentés).

Même si les codes d'emploi étaient fondés sur l'utilisation d'une méthode uniforme, la subjectivité du codeur peut avoir entraîné une classification erronée. Bien que nous n’ayons pas effectué de validation officielle, nous avons observé les tendances attendues entre les groupes d'emploi et au sein de ceux-ci pour ce qui est du sexe, de l'âge et de la disponibilité des emplois. Par exemple, les consultations aux SU pour les accidents de travail des livreurs en pourcentage de l'ensemble des consultations aux SU liées au travail ont baissé, passant de 13,5\% entre 1991 et 1997 à 4,4 \% entre 2005 et 2012, ce qui coïncide probablement avec la diminution de la circulation des journaux imprimés. Les RPB pour les dossiers « impossibles à déterminer » étaient généralement similaires aux RPB regroupés, ce qui indique que les dossiers ambigus n'étaient pas liés à un groupe d'emploi ou à un secteur particulier, et les RPB ont aussi fait état de caractéristiques de blessures prévisibles en fonction du groupe d'emploi, montrant la validité préliminaire de nos classifications. À la lumière de ces signes, une classification erronée systématique du codage des emplois semble peu plausible.

\section{Conclusion}

Nous avons utilisé un mécanisme de classification nationale des professions normalisé ainsi que des RPB pour analyser les blessures liées au travail chez les jeunes enregistrées par le système de surveillance du SCHIRPT. Cette étude s'ajoute au corpus croissant de données probantes indiquant une incidence supérieure de blessures spécifiques dans les groupes d'emploi et en fonction de l'âge par rapport aux blessures non professionnelles. Ces conclusions peuvent servir à soutenir l'adoption de mesures de prévention ciblées et en fonction de l'âge susceptibles de permettre une réduction du nombre de blessures.

\section{Références}

1. Wray-Lake L, Syvertsen AK, Briddell L, Osgood DW, Flanagan CA. Exploring the changing meaning of work for American high school seniors from 1976 to 2005 . Youth Soc. 2011;43(3):1110-1135.

2. Statistique Canada. Tableau 282-0087. Enquête sur la population active (EPA), estimations selon le sexe et le groupe d'âge, désaisonnalisées et non désaisonnalisées, mensuel (personnes sauf indication contraire), CANSIM (base de données), 2015 [Internet]. Ottawa (Ont.) : Statistique Canada; 2015 [consultation le 27 mars 2015]. Consultable en ligne à la page; http://www5.statcan.gc.ca/cansim/a26? id $=2820087$ \& retrLang $=$ fra\&lang $=$ fra 
3. Association des commissions des accidents du travail du Canada. Programme national de statistiques sur les accidents du travail (PNSAT). Tableau 24. Nombre de décès, par groupe d'âge et par province/territoire, 2011-2013. Toronto (Ont.) : ACATC; 2013.

4. Association des commissions des accidents du travail du Canada. Programme national de statistiques sur les accidents du travail (PNSAT). Tableau 3. Nombre d'accidents acceptés avec perte de temps, par groupe d'âge et par province/territoire, 2011-2013. Toronto (Ont.) : ACATC; 2013.

5. Zierold KM, Garman S, Anderson H. Summer work and injury among middle school students, aged 10-14 Years. Occup Environ Med. 2004;61(6):518-522.

6. Barnetson B. Incidence of work and workplace injury among Alberta teens. Can J Work Soc. 2013;20(Summer):14-32.

7. Breslin C, Koehoorn M, Smith P, Manno M. Age related differences in work injuries and permanent impairment: a comparison of workers' compensation claims among adolescents, young adults, and adults. Occup Environ Med. 2003;60(9):E10.

8. Koehoorn M, Breslin FC, Xu F. Investigating the longer-term health consequences of work-related injuries among youth. J Adolesc Health. 2008;43(5):466-73.

9. Breslin FC, Pole JD, Tompa E, Amick BC, Smith $\mathrm{P}$, Johnson SH. Antecedents of work disability absence among young people: a prospective study. Ann Epidemiol. 2007;17(10):814-820.

10. Galarneau D. L'écart salarial entre employés temporaires et permanents. L'emploi et le revenu en perspective. 2005;17(1):40-53

11. Lewko J, Hall K, Egeh G, Volpe R, Fuhringer $\mathrm{C}$, Tremblay C. Current health and safety policies protecting young workers in Canada, 2011. Sudbury (Ont.) : Université Laurentienne; 2011 Publication conjointe de l'Ontario Neurotrauma Foundation.

12. Loughlin C, Barling J. Young workers' work values, attitudes, and behaviours. J Occup Organ Psychol. 2001;74(4):543-558. DOI : 10.1348/096317901167514.

13. Tucker S, Diekrager D, Turner N, Kelloway EK. Work-related injury underreporting among young workers: prevalence, gender differences, and explanations for underreporting. J Safety Res. 2014;50:67-73.
14. Curtis Breslin F, Polzer J, MacEachen E, Morrongiello B, Shannon H. Workplace injury or "part of the job"?: Towards a gendered understanding of injuries and complaints among young workers. Soc Sci Med.2007;64(4):782-793.

15. Lipskie T, Breslin FC. A descriptive analysis of Canadian youth treated in emergency departments for work-related injuries. Chronic Dis Can. 2005;26(4):107-113.

16. Macarthur C, Pless IB. Evaluation of the quality of an injury surveillance system. Am J Epidemiol. 1999;149(6):586-592.

17. Statistique Canada. Ministre de l'Industrie. Classification nationale des professions pour statistiques (CNP-S) 2006. Ottawa (Ont.) : Statistique Canada; 2007. [Statistique Canada, Catalogue $\left.\mathrm{N}^{\mathrm{o}} 12: 583-\mathrm{XIF}\right]$.

18. Barell V, Aharonson-Daniel L, Fingerhut LA, Mackenzie EJ, Ziv A, Boyko V et collab. An introduction to the Barell body region by nature of injury diagnosis matrix. Inj Prev. 2002;8(2):91-96.

19. Bhattacharya A. Costs of occupational musculoskeletal disorders (MSDs) in the United States. Int J Ind Ergon. 2014;44 (3):448-454. DOI: 10.1016/j.ergon.2014 01.008 .

20. Boyle P, Parkin DM. Chapitre 11. Les registres hospitaliers de cancer. Sous la direction deJensen OM, Parkin DM, MacLennan R, Muir CS, Skeet RG) (Enregistrement de cancers : principes et méthodes. Lyon (France) : Centre international de recherche sur le cancer; 1991 p. 126.

21. Breslow NE, Day NE. Statistical methods in cancer research. Volume II-The design and analysis of cohort studies. IARC Scientific Publication $n^{\circ}$ 82. Lyon (France) : Centre international de recherche sur le cancer; 1987.

22. Do MT, Fréchette M, McFaull S, Denning B, Ruta M, Thompson W. Injuries in the North-analysis of 20 years of surveillance data collected by the Canadian Hospitals Injury Reporting and Prevention Program. Int J Circumpolar Health. 2013;72:1-6.

23. Deits J, Yard EE, Collins CL, Fields SK, Comstock RD. Patients with ice hockey injuries presenting to US emergency departments, 1990-2006. J Athl Train. 2010;45(5):467-474. DOI: $10.4085 / 1062-6050-45.5 .467$.
24. Lipscomb HJ, Li L. Injuries among teens employed in the homebuilding industry in North Carolina. Inj Prev. 2001;7(3):205-209.

25. Knowles SB, Kucera KL, Marshall SW. Commentary: the injury proportion ratio: what's it all about? J Athl Train. 2010;45(5) 475-277.

26. SAS Institute Inc., Cary, NC, USA. SAS software, SAS system for Windows. Copyright (c) 2002-2012. 20129.4.

27. Reichard AA, Konda S, Jackson LL. Occupational burns treated in emergency departments. Am J Ind Med. 2015;58(3): 290-298. DOI: 10.1002/ajim.22407.

28. Runyan CW, Vladutiu CJ, Rauscher KJ, Schulman M. Teen workers' exposures to occupational hazards and use of personal protective equipment. Am J Ind Med. 2008; 51(10):735-740. DOI: 10.1002/ajim.20624.

29. Welch L, Hunting K, Mawudeku A. Injury surveillance in construction: eye injuries. Appl Occup Environ Hyg. 2001;16(7): 755-762.

30. Lombardi DA, Verma SK, Brennan MJ, Perry MJ. Factors influencing worker use of personal protective eyewear. Accid Anal Prev. 2009;41(4):755-762.

31. Breslin FC, Ibrahim S, Smith P, Mustard C, Amick B, Shankardass K. The demographic and contextual correlates of work-related repetitive strain injuries among Canadian men and women. Am J Ind Med. 2013;56(10): 1180-1189.

32. Harvey HH. Reducing traumatic brain injuries in youth sports: youth sports traumatic brain injury state laws, January 2009December 2012. Am J Public Health. 2013; 103(7):1249-1254. DOI: 10.2105/AJPH.2012.3 01107.

33. Anderson V, Brown S, Newitt H, Hoile H. Educational, vocational, psychosocial, and quality-of-life outcomes for adult survivors of childhood traumatic brain injury. J Head Trauma Rehabil. 2009;24(5):303-312. DOI: 10.1097/HTR.0b013e3181ada830.

34. Statistique Canada. Enquête sur la population active (EPA) [Internet]. Ottawa (Ont.) : Statistique Canada; 2015 [consultation le 16 avril 2015]. Consultable en ligne à la page : http://www23.statcan.gc.ca/imdb/p2SV_ f.pl?Function $=$ getSurvey\&SDDS $=3701$ 
35. Pickett W, Brison RJ, Mackenzie SG et collab. Youth injury data in the Canadian Hospitals Injury Reporting and Prevention Program: do they represent the Canadian experience?. Inj Prev. 2000;6(1):9-15.

36. Kang J, Hagel B, Emery CA, Senger T, Meeuwisse W. Assessing the representativeness of Canadian Hospitals Injury Reporting and Prevention Programme (CHIRPP) sport and recreational injury data in Calgary, Canada. Int J Inj Contr Saf Promot. 2013;20(1):19.

37. Institut canadien d'information sur la santé. Statistiques sur les cas de blessures et de traumatismes au service d'urgence, 2013-2014 [Internet]. Ottawa (Ont.) : ICIS; 2015 [consultation le 22 octobre 2015]. Consultable en ligne à la page; https://www.cihi.ca/fr/ types-de-soins/services-specialises/statistiques-sur-les-cas-de-blessures-et-de-traumatismes-au 